\title{
Multi-Machine Power Stabilization Controller (MMPSC) for Power Quality Applications
}

\author{
D. Sabapathi' ${ }^{1}$ R. Anita ${ }^{2}$ \\ ${ }^{1}$ Department of Electrical and Electronics, MPNMJ Engg. College, Chennimalai, Erode, India \\ ${ }^{2}$ Department of Electrical and Electronics, IRTT, Erode, India \\ Email: esec74@yahoo.co.in, anita_irtt@yahoo.co.in
}

Received 28 April 2016; accepted 15 May 2016; published 12 August 2016

Copyright (C) 2016 by authors and Scientific Research Publishing Inc.

This work is licensed under the Creative Commons Attribution International License (CC BY).

http://creativecommons.org/licenses/by/4.0/

(c) (i) Open Access

\section{Abstract}

Power system stability control is a challenging task in power generation, transmission and distributions based applications and in many fields. Multi-machine power compensation control can achieve system stabilization within a prescribed time in conventional controller. However, limited time control cannot guarantee the system convergence within particular time independent on the initial condition, which makes illegal application into the practical system if the initial condition is unknown in advance. The proposed Multi-Machine Power System Compensation (MMPSC) control overcomes the issues in existing systems and limited time stability controller. Due to this attractive solution, multi-machine power compensation control stability has found applications in uniform exact differentiator design for the multi-agent system. The proposed multi-machine power compensation control reduces damping oscillation and improves the power system stability control. The main objective of proposed controller is to improve the stability of MMPSC limited time system stabilization independent of the initial state and ensure fast convergence both far away from and at a close range of the power monitoring system. This feature can reduce the loss caused by unwanted oscillation and avoid voltage collapse. To overcome the linearity problem of terminal mode control, saturation function is introduced to limit the amplitude of power input. In comparison with the existing results on stability control, the proposed MMPSC applies a simpler method to overcome stability problem and achieves higher efficiency.

\section{Keywords}

POD, PSS, CPSS, MMPSC, DFIG and MATLAB 


\section{Introduction}

Power systems unit is classified into three different stages namely generation, transmission, and distribution. In the generation unit, the electric power is generated mostly by using synchronous generators. Transformers are used to increase the power and reduce the line currents which automatically reduce the power transmission losses. It is necessary to maintain the power systems with continuous power supply and voltage stability. However, the unpredictable events such as lightning, accidents or any other critical situation, short circuits between the transmission lines produce a fault. The power system fault produce, one or more generators, may be severely disturbed causing an imbalance between generation and demand. If the failure persists and not cleared in a pre-specified timeframe, it may cause severe damages to the equipment which in turn may lead to a power loss and electricity outage.

The electric power demand should be satisfied in a reliable, stable and quality power in sensitive power industries like information technology, communication, electronics, etc. The power system engineers to provide a stable and adequate power quality parameter to the consumers. In this work, we discussed the access of stability in a power system, improvement of the stability and prevention methods of the unstable system. In this study, we have used different methods for stabilization under various disturbances in the wind farm. Researchers have not given attention to proper wind farm stabilization in some previous researches. Recently, the most widely used variable wind turbine is the Doubly Fed Induction Generator type (DFIG) because it can operate at a wider range of speed depending on the wind speed or other specific operation requirements. Hence, this work explains about the improvement in power quality and stability analysis performed on FACTS devices for flexible MMPSC power control damping of the power system and stabilization of power system. This work presents a comparative analysis of the use of multi-machine power system stabilization.

From the above discussion, this work proposes a time constrained MMPSC method and applies it to design energy storage device controller for noise reduction in power system. The main advantage of proposed controller is that it can guarantee finite time system stabilization independent on initial state and ensure fast convergence both far away from and at a close range of the control objective. This feature can reduce the loss caused by instant oscillation and avoid voltage collapse. In order to overcome the singularity problem of load voltage control, saturation function is introduced to limit the amplitude of control input. In comparison with the existing results such as Automatic Voltage Regulator (AVR) and Artificial Neural Network (ANN) on fixed-time control, the proposed controller applies a simpler method to overcome singularity problem and achieves faster convergence.

Hence the proposed work implementation based on closed loop system stability analysis involved in the system models has been presented. The open loop system is unstable, and three damping controllers are implemented in the multi-machine system to enhance the system stability. A detailed stability analysis has been presented, based on the computed eigen values, damping ratios and deviation responses of the system models. Based on the various closed loop stability analysis, it is evident that the proposed MMPSC is better than the conventional AVR and ANN based controller for multi-machine power system stability enhancement.

\section{Literature Review}

Numerous related research works are already existed in literature which based on power system stability and power quality control system. Some of them are reviewed here.

Power system is a complex nonlinear dynamical system and when the power system operates near its stability boundary, parameter variations [1], time delay [2] and external disturbances [3] can induce chaos. Chaotic oscillation is an undesirable phenomenon for power system and it may result

in voltage collapse [4] [5] and even catastrophic blackouts [6]. Therefore, it is necessary to study chaos control method to avoid voltage collapse in power system. Different control schemes have been proposed for chaos suppression and voltage stabilization, such as, parameter perturbation method [7], washout filter aided feedback [8], feedback linearization [9], ANFIS based control [10], adaptive control using LaSalle's invariance principle [11], etc. However, all the aforementioned control methods can only achieve asymptotic stability, that is, the convergence time cannot be assigned in advance. From the viewpoint of power system operation, oscillations are acceptable if they can be damped within a limited time.

Finite time control can achieve system stabilization within a prescribed time and it has been applied in many fields (for instance, [12] [13]). However, finite time control cannot guarantee the system convergence within 
bounded time independent on the initial condition, which prohibits its application into practical system if theinitial condition is unknown in advance.

The fixed-time stability introduced by Polyakov [14] can overcome this drawback. Due to this attractive property, fixed-time stability has found applications in uniform exact differentiator design [15] and consensus for multi-agent system [16]. However, there are few results about fixed-time stable control. Zuo [17] proposed a non-singular fixed-time terminal sliding mode controller for a class of second order nonlinear systems. However, the convergence time of the non-singular fixed-time terminal sliding mode controller is not an optimal one; moreover, the method used to eliminate singularity is complicated.

The high penetration of DFIG wind farms in power systems may adversely impact on the damping of oscillation modes due to the reduction of system inertia, the interaction among DFIG converter controls, the less synchronized coupling, and the displacement of synchronous generators (SGs) [18]. These situations cause the complicated power oscillations problem which may lead to wide area blackouts. Under these scenarios, the conventional power system stabilizer (PSS) may not be able to handle the power oscillations [19].

To augment the PSS effect, the coordinated power oscillation damper (POD) is significantly anticipated. Recently, the DFIG wind turbine which is equipped with the POD has been applied to suppress the power oscillations based on the active and reactive output power control of the DFIG [20]. This makes the possibility of the coordinated control of POD and PSS for the stabilization of power oscillations. In [21], the optimized partial eigen-structure assignment for the design of the combined PSS and POD is proposed. Additionally, the coordinated POD and PSS considering output power variation is presented in [22].

From the above issues, an innovative work is needed to ignore the fact that voltage instability always accompanies with frequency oscillation caused by active power imbalance. In power system, energy storage device and adaptable compensator can provide flexible active and reactive power compensation respectively. Therefore, design of proposed controller and energy storage device controller to achieve system stabilization within a limited time is significant for chaos suppression in power system.Hence the work concentrates to develop techniques which significantly improve the stability analysis with better efficiency and high power quality between Grid systems with acceptable delay.

\section{Multi-Machine Power System Compensation Controller (MMPSC) Methodology}

The general power system architecture for voltage stability model is shown in Figure 1. MMPSC scheme is applied to design MMPSC controller and energy storage device controller for voltage stability improvement in power system. The power system model consists of two generator buses and one load bus. One generator bus is treated as a slack bus, while the other generator is equipped with energy storage device and its dynamics can be described by stability equation. The load bus includes a dynamic induction motor in parallel with a constant PQ load and a MMPSC. The mathematical model for the dynamic motor, MMPSC and energy storage device controller are described in the section of mathematical formulation.

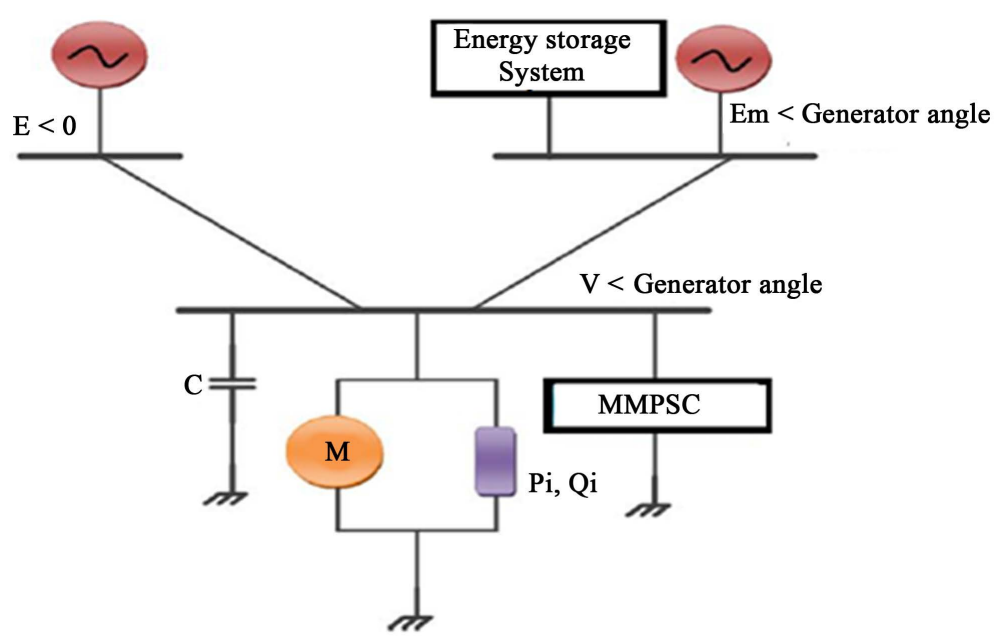

Figure 1. MMPSC system model. 
Multi-machine power compensation control can achieve system stabilization within a prescribed time, and it has been applied in many fields. However, limited time control cannot guarantee the system convergence within particular time independent on the initial condition, which makes illegal application into the practical system if the initial condition is unknown in advance. The proposed multi-machine power compensation control overcomes the issues in existing systems and limited time stability controller. Due to this attractive solution, MMPSC stability has found applications in uniform exact differentiator design for the multi-agent system. However, MMPSC proposed a nonlinear time terminal mode controller for a class of second-order nonlinear systems. However, the proposed method eliminates the convergence time of the multi-machine controller is provides an optimal solution and also it is used to remove system complications.

\section{Modelling of MMPSC Controller}

The mathematical model for the dynamic motor, MMPSC and energy storage device controller are taken from following equations. The dynamics of controlled power system model can be described as: The inequality holds along every trail of the multi-machine power system. The proposed work revises the following statement. Consider a transmission network interconnects a multi-machine power system satisfying Assumption 1 with $\mathrm{N}$ generators described by following Equations and $\mathrm{M}$ loads.

Let $\varepsilon^{*}$ be an equilibrium point for the generators that is consistent with all the equations describing the power system. The equilibrium $\varepsilon^{*}$ is globally asymptotically stable if

$$
\left(L_{s s, i} I_{x, i}^{*}\right)^{2}+\left(L_{s s, i} I_{y, i}^{*}\right)^{2}<4 D_{i} r_{i}
$$

holds for all $i\{f 1 \ldots . . . . . \mathrm{Ng}\}$.

However, we can design a control law, where a MMPSC compensator device enforces a voltage drop of

$$
\left(T_{\theta i}^{T}-T_{\theta i^{*}}^{T}\right) V_{a b c, I}^{*}
$$

and a MMPSC device enforces a current drop of at the terminals of the $i^{\text {th }}$ generator,

$$
\left(T_{\theta i}^{T}-T_{\theta i^{*}}^{T}\right) I_{a b c, I}^{*}
$$

where $\theta_{i}$ is the rotor angle of the $i^{\text {th }}$ generator, $\theta_{i^{*}}$ is the reference rotor angle of the same generator and $T_{\theta}$ is defined

$$
T_{\theta}=\frac{\sqrt{2}}{3}\left[\begin{array}{cccc}
\cos (\theta) & \cos \left(\theta-\frac{2 \pi}{3}\right) & \cos \left(\theta+\frac{2 \pi}{3}\right) & 0 \\
\sin (\theta) & \sin \left(\theta-\frac{2 \pi}{3}\right) & \sin \left(\theta+\frac{2 \pi}{3}\right) & 0 \\
\frac{\sqrt{2}}{2} & \frac{\sqrt{2}}{2} & \frac{\sqrt{2}}{2} & 0
\end{array}\right]
$$

We note that $T_{\theta}^{-1}=T_{\theta}^{T}$. Typically, steady state angle differences between the generators are provided, and the reference rotor angles can be constructed a priori from this information by choosing one of the regular state angles as the reference frame. Under this control law, the voltages and currents in XYZ coordinates at the $i^{\text {th }}$ generator terminals become

$$
\begin{gathered}
V_{x y z, i, \text { new }}=V_{x y z, i}-\left(T_{\theta i}^{T}-T_{\theta i^{*}}^{T}\right) V_{a b c, i}^{*}=T_{\theta i}^{T} V_{a b c, i} \\
I_{x y z, i, \text { new }}=I_{x y z, i}-\left(T_{\theta i}^{T}-T_{\theta i^{*}}^{T}\right) I_{a b c, i}^{*}=T_{\theta i}^{T} I_{a b c, i}
\end{gathered}
$$

Equations (5)-(6) show that by applying voltage drop and current drop , we can equalize the incremental power in xyz and abc coordinates, i.e.,

$$
V_{x y z, i, \text { new }}^{T} I_{x y z, i, \text { new }}=V_{a b c, i}^{T} T_{\theta i} T_{\theta i}^{T} I_{a b c, i}=V_{a b c, i}^{T} I_{a b c}
$$

We now show that, under the action of these controllers, multi-machine power compensation control (MMPSC) is always satisfied. 


\section{Mathematical Formulation}

The primary objective of any compensation scheme is that it should have a fast response, flexible and easy to implement. The control algorithms of an MMPSC mainly applied in the following steps:

1. Measurements of system voltages and current,

2. Signal conditioning,

3. Calculation of compensating signals.

The control block (Figure 2) used which switch Pure Wave MMPSC modules as required to control the external devices such as mechanically switched capacitor banks. The performance of the MMPSC depends on the PQ Theory algorithm i.e. the extraction of the current components. The MMPSC control performance is analyzed and verified using power device which provides fast response, suitable for dynamic load response or voltage regulation and automation needs. MMPSC also used to correct voltage swells or sags caused by reactive power demands on a wide range of distribution and transmission voltage, overload capability of this provides reserve energy for transients

The three-phase unbalanced load currents in the a-b-c reference frame can be expressed as follows. A radial distribution system with an unbalance load and a MMPSC

$$
\begin{gathered}
{\left[\begin{array}{c}
\tilde{V}_{a b}^{L} \\
\tilde{V}_{b c}^{L} \\
\tilde{V}_{c a}^{L}
\end{array}\right]=\left[\begin{array}{ccc}
1 & 1 & 1 \\
1 & \alpha^{2} & \alpha \\
1 & \alpha & \alpha^{2}
\end{array}\right]\left[\begin{array}{c}
\tilde{V}_{u, 0}^{L} \\
\tilde{V}_{u, 1}^{L} \\
\tilde{V}_{u, 2}^{L}
\end{array}\right]=[T]\left[\begin{array}{c}
\tilde{V}_{u, 0}^{L} \\
\tilde{V}_{u, 1}^{L} \\
\tilde{V}_{u, 2}^{L}
\end{array}\right]} \\
{\left[\begin{array}{c}
\bar{I}_{a}^{L} \\
\bar{I}_{b}^{L} \\
\bar{I}_{c}^{L}
\end{array}\right]=\left[\begin{array}{ccc}
Y_{a b}^{L} & 0 & -Y_{c a}^{L} \\
-Y_{a b}^{L} & Y_{b c}^{L} & 0 \\
0 & -Y_{b c}^{L} & Y_{c a}^{L}
\end{array}\right]\left[\begin{array}{c}
\tilde{V}_{a b}^{L} \\
\tilde{V}_{b c}^{L} \\
\tilde{V}_{c a}^{L}
\end{array}\right]}
\end{gathered}
$$

Applying the symmetrical components method transfers the three-phase load currents to positive- and negative-sequence components, as shown, the linnet- line voltages are assumed equal to simplify the derivation of the compensation scheme.

$$
\begin{aligned}
& \bar{I}_{1}^{L}=(1-\alpha) Y_{0}^{L} V_{u}^{L} \\
& \bar{I}_{2}^{L}=(1-\alpha) Y_{1}^{L} V_{u}^{L}
\end{aligned}
$$

where: $\left[\begin{array}{l}Y_{0}^{L} \\ Y_{1}^{L} \\ Y_{2}^{L}\end{array}\right]=\frac{1}{3}\left[\begin{array}{ccc}1 & 1 & 1 \\ 1 & \alpha^{2} & \alpha \\ 1 & \alpha & \alpha^{2}\end{array}\right]\left[\begin{array}{c}Y_{a b}^{L} \\ Y_{b c}^{L} \\ Y_{c a}^{L}\end{array}\right], \quad V_{l l}^{L}=\left|\tilde{V}_{l l, 1}^{L}\right|$.

The detection of the load power can be obtained via two wattmeter method, as shown in Equation (10), the

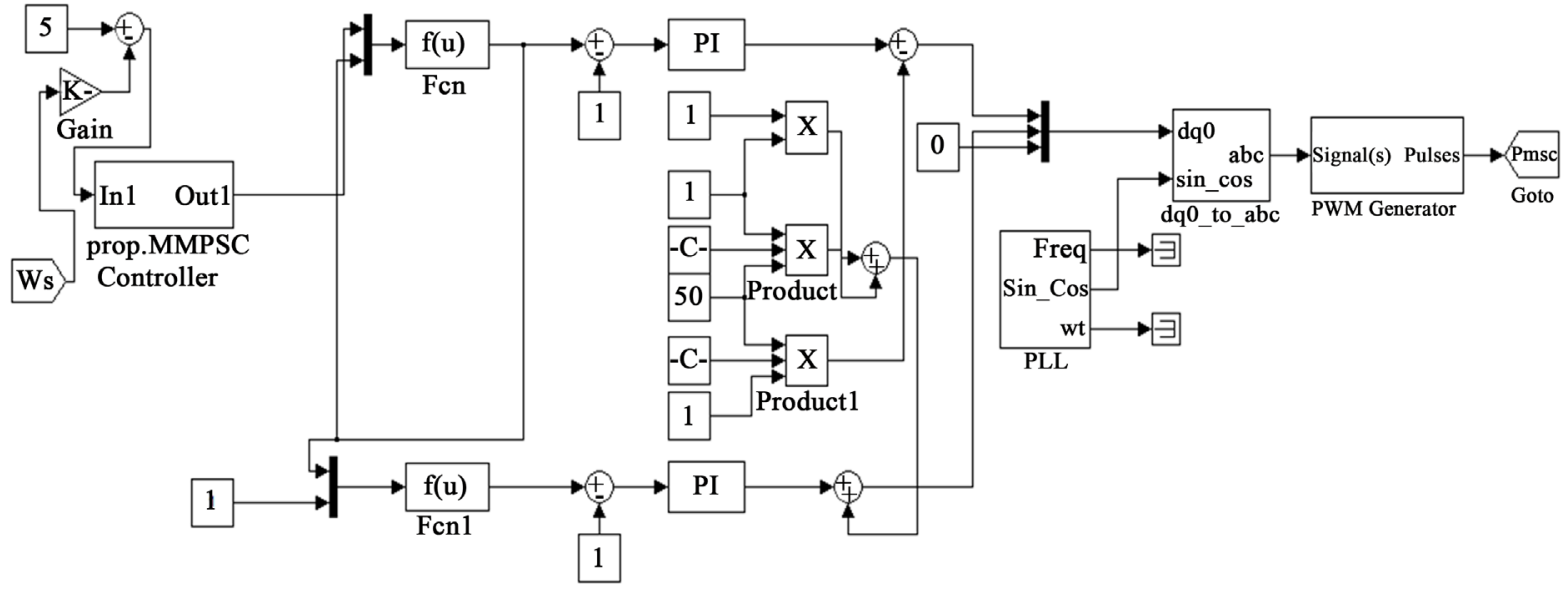

Figure 2. Control block of MMPSC. 
positive- and negative-sequence load currents are represented with line-to-line active and reactive powers, as shown Equation (11)

$$
\begin{gathered}
\left(\tilde{V}_{a b}^{L}\right)^{*}\left(\bar{I}_{a}^{L}\right)=\left(V_{l l}^{L} \angle-30^{\circ}\right)\left[\left(\bar{I}_{1}^{L}\right)+\left(\bar{I}_{2}^{L}\right)\right] \\
\left(\tilde{V}_{a b}^{L}\right)^{*}\left(\bar{I}_{c}^{L}\right)=\left(V_{l l}^{L} \angle-90^{\circ}\right)\left[\alpha\left(\bar{I}_{1}^{L}\right)+\alpha^{2}\left(\bar{I}_{2}^{L}\right)\right] \\
{\left[\begin{array}{c}
\bar{I}_{1}^{L} \\
\bar{I}_{2}^{L}
\end{array}\right]=\frac{1}{\left|V_{l l}^{L}\right|(\alpha-1)}\left[\begin{array}{cc}
1 \angle 150^{\circ} & 1 \angle 150^{\circ} \\
1 \angle-150^{\circ} & 1 \angle-30^{\circ}
\end{array}\right]\left[\begin{array}{ll}
P_{a b}^{L} & -j Q_{a b}^{L} \\
P_{c b}^{L} & -J Q_{a b}^{L}
\end{array}\right]}
\end{gathered}
$$

For fast load compensation, the MMPSC should compensate the imaginary part of the positive-sequence load current and the entire negative-sequence load current in (11) as soon as possible. In this way, the power source supplies only real part of the positive-sequence load current.

Since no zero sequence component appears in three-phase three-wire system, the compensation current can be derived from Equation (10) (11). Finally, the needed compensation current of the MMPSC for load compensation is obtained, as shown

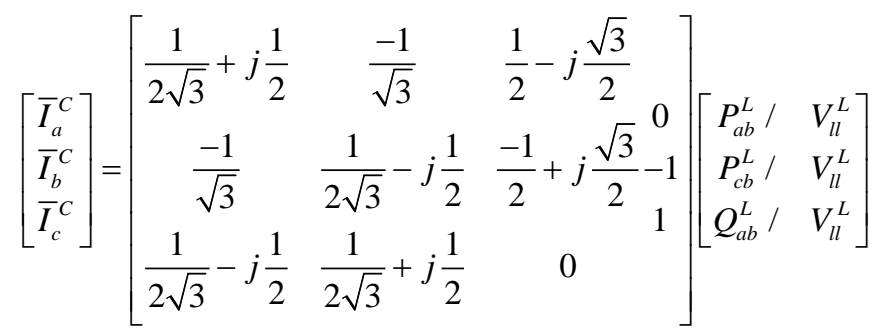

$$
\begin{aligned}
& \bar{I}_{a}^{c}=\operatorname{Im}\left(\bar{I}_{1}^{L}\right)+\bar{I}_{2}^{L}, \bar{I}_{b}^{c}=\alpha^{2} \operatorname{Im}\left(\bar{I}_{1}^{L}\right)+\alpha \bar{I}_{2}^{L}, \bar{I}_{c}^{c}=\alpha \operatorname{Im}\left(\bar{I}_{1}^{L}\right)+\alpha^{2} \bar{I}_{2}^{L}
\end{aligned}
$$

The overall compensation scheme of the MMPSC is now completed

$$
\left|I_{r}\right|=K_{P} \Delta v_{d c}+K_{i} \int \Delta v_{d c} \mathrm{~d} t
$$

For fast real-time compensation, the MMPSC needs to detect the line-to-line power data very quickly to calculate the required compensation current, as shown in (14). With a high-performance DSP-based system, the compensation scheme and other necessary functions regarding power detections can be implemented very quickly. In this way, the necessity for measuring instruments is reduced. This significantly reduces the constructing cost of the MMPSC and enhances the system reliability (Figure 3).

\section{Results and Discussion}

All the above discussed approaches have been implemented in Matlab tool, with various scenarios of multi-machine systems under different fault conditions. Each method has been tested for its voltage stability improvement in number of factors such as frequency, speed deviation, Power angle deviation and time duration under scenarios. The performance of the proposed MMPSC method is tested with available on IEEE Bus systems. The efficiency of the proposed model has been evaluated as follows.

\subsection{IEEE Test System}

Test system described to implement a two IEEE test systems namely two-area test system and 16-machine, a 68-bus system for identification of inter-area oscillations. The proposed method also validated on real time signals captured by WAFMS at Indian Institute of Technology, Mumbai, India.

A benchmark model consisting of 16-machine, 68-bus system shown in Figure 4 represents five geographical regions with tie-lines highlighted by a thick line. This proposed system a reduced order equivalent of the interconnected New England test system (NETS) and New York power system (NYPS). The first nine machines represent NETS generation, while represent NYPS generation. The last three machines represent three other neighbouring areas which are approximated by their equivalent generator models. The detailed description of the 


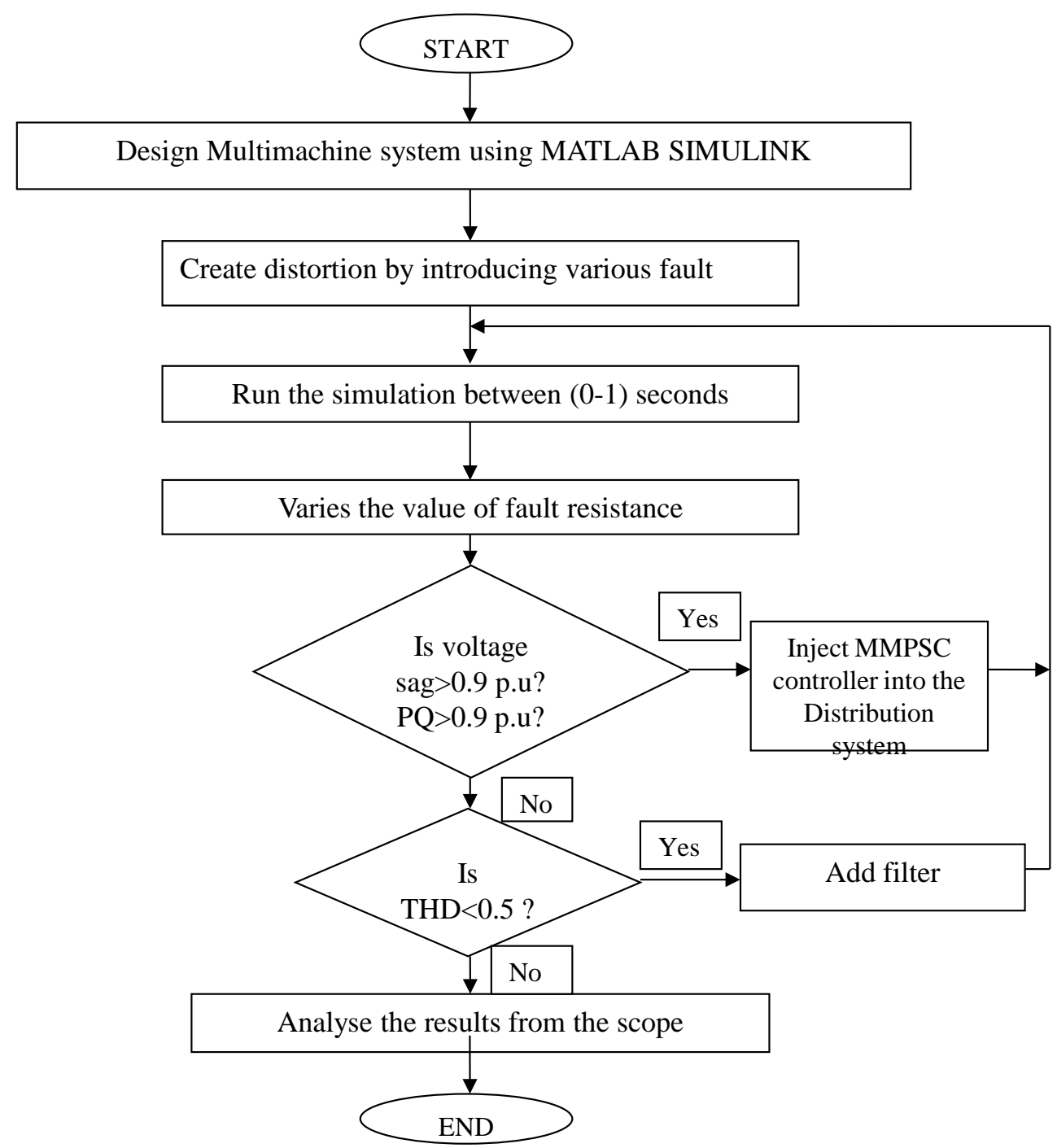

Figure 3. Flowchart of MMPSC design methodology.

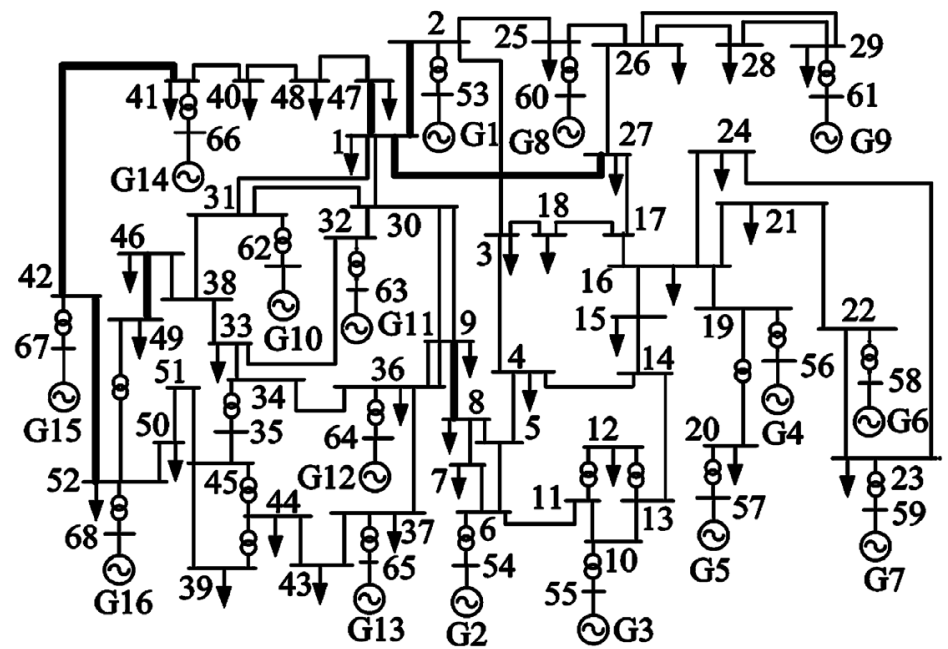

Figure 4. Single line diagram of 16-machine 68-bus system. 
test system along with network, devices and bus data are available. All machines have static exciters with PSS installed on all machines except for generators 13, 14, 15 and 16. Loads are modeled as constant impedance type. For the present work, tie-lines 46 - 49 and 1 - 47 are assumed to be out of commission.

\subsection{Parameter Selection of IEEE Test System}

The Generator angle, frequency deviation and load voltage are depends on the number of Generators participation in generation. Table 1 shows the detail presents the frequency and damping of four inter-area modes present in the system. The generators participating in each mode are also given.

\subsection{Multi-Machine System Oscillations Damping Analysis}

Implementation of the proposed MMPSC controllers in multi-machine model provides the optimal controller parameters. The MMPSC system damping analysis oscillations in the form of rotor speed and power angle deviations are represented in Figures 5-8 for generators G2 and G3 for various operating conditions and disturbances. The experimental oscillations for all operating conditions in non linear time domain based MMPSC results are as follows.

The above results show the dynamic responses point out the deviation overshoots are minimized and damped as early as possible for the various controllers (AVR, ANN and MMPSC). The better damping oscillation $\mathrm{P}=$ $0.33, \mathrm{Q}=0.03, \mathrm{Pd}=0.02$ p.u was shown in Figure 5, the maximum speed deviation overshoot obtained for Automatic voltage regulator (AVR) is $4.2 \times 10^{-4} \mathrm{p}$.u, for Artificial neural network(ANN) is $3 \times 10^{-4}$ and for Proposed it is $2.6 \times 10^{-4}$ p.u.

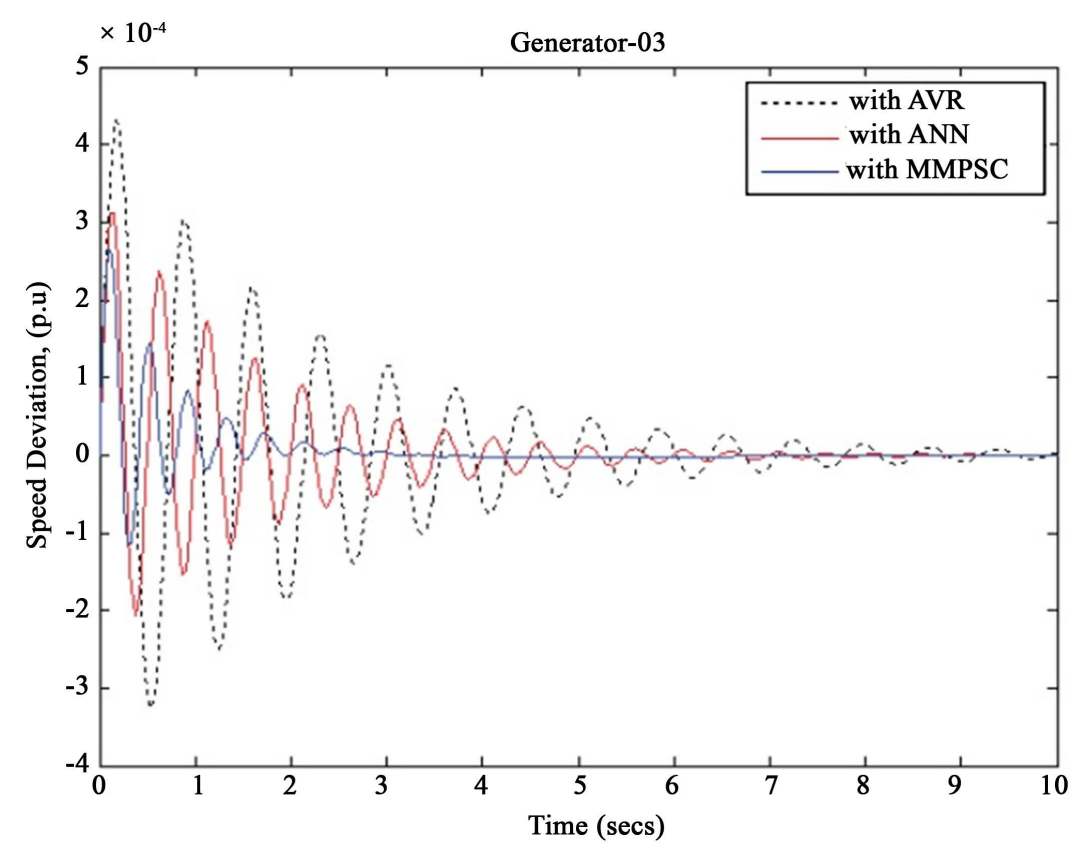

Figure 5. Speed deviation responses of G3 for $\mathrm{P}=0.33, \mathrm{Q}=0.03, \Delta \mathrm{Pd}=0.02 \mathrm{p}$. u condition.

Table 1. Stability analysis parameters under different mode of IEEE 16 bus system.

\begin{tabular}{cccr}
\hline Modes & Frequency $(\mathrm{Hz})$ & Damping value & Generators participating \\
1 & 0.2199 & 0.2366 & G2-G7, G9, G12-G14, G16 \\
2 & 0.4382 & 0.0939 & G14, G16 \\
3 & 0.6613 & 0.0885 & G3-G7, G9, G12, G13 \\
4 & 0.7727 & 0.0859 & G14, G15, G16 \\
\hline
\end{tabular}




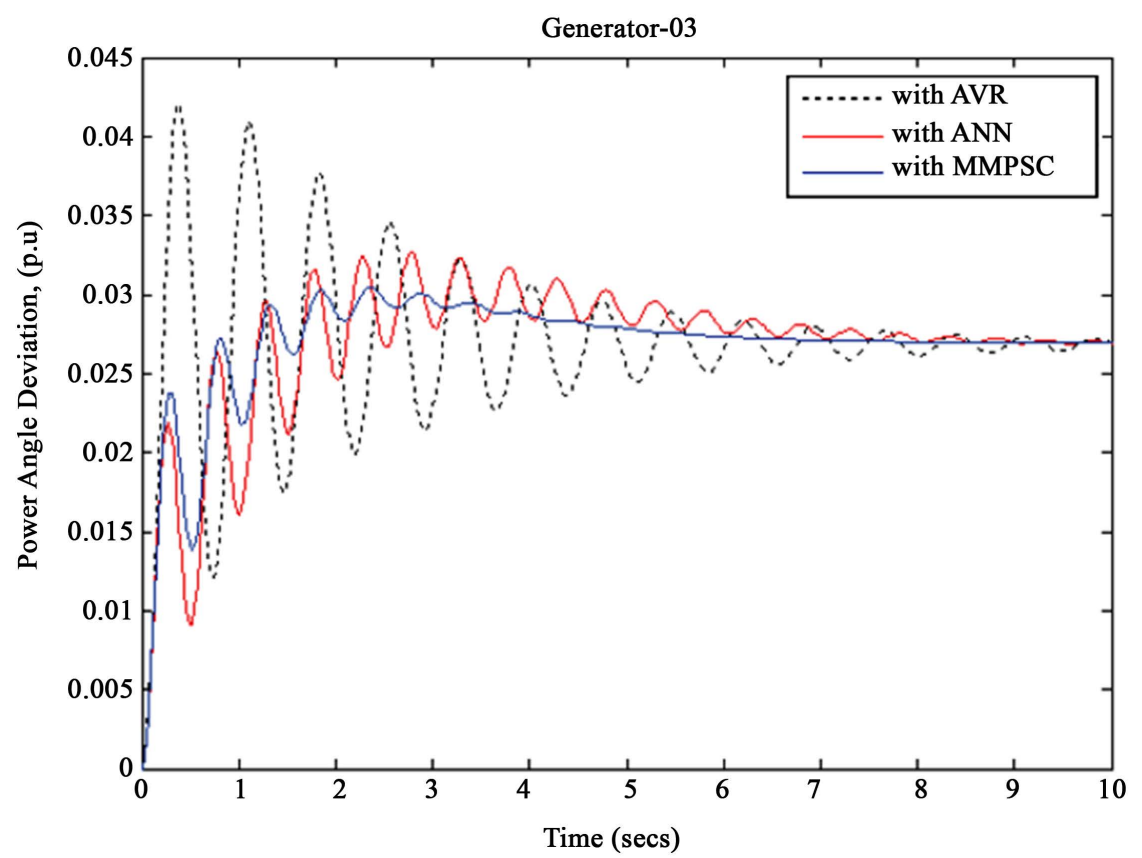

Figure 6. Power angle deviation responses of $\mathrm{G} 3$ for $\mathrm{P}=0.24, \mathrm{Q}=0.055, \Delta \mathrm{Pd}=0.02 \mathrm{p} . \mathrm{u}$ condition.

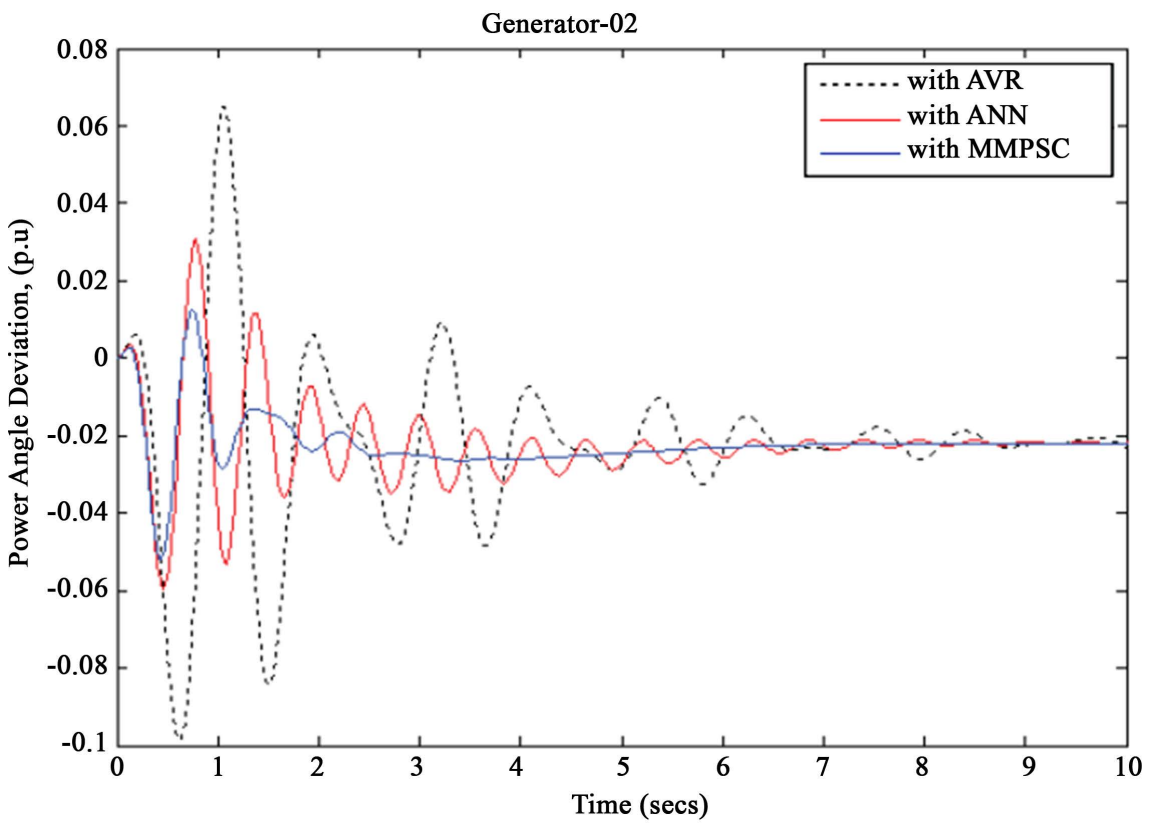

Figure 7. Power angle deviation responses of $\mathrm{G} 2$ for $\mathrm{P}=0.61, \mathrm{Q}=0.051, \Delta \mathrm{Pd}=0.03 \mathrm{p}$.u condition.

\subsection{Comparison for New England Test System}

The proposed approach employs a MMPSC control technique to search optimal design of multi-machine power system stabilizers. Table 2 gives the comparison of performance of AVR, ANN and MMPSC for NETS based IEEE System under heavy loading condition. From above results show the dynamic responses point out the deviation overshoots are minimized and damped as early as possible for the various controllers (AVR, ANN and MMPSC). The better damping ratio for various controllers are observed and noted in Table 2. AVR is $\mathbf{0 . 1 7 8 4 5}$ p.u, for Artificial Neural Network(ANN) is $\mathbf{0 . 2 3 1 0 1}$ p.u and for Proposed it is $\mathbf{0 . 6 2 4 3 3 5}$ p.u. Also the deviations 
settle at 9 sec AVR, 6 sec ANN and only 3 seconds for Proposed MMPSC. This shows the effective damping exerted by the proposed MMPSC based controller design. These time domain responses clearly confirm the damping action and stability enhancement provided by the proposed MMPSC controllers for various operating conditions of the wind system considered for analysis.

Figure 9 shows the comparative charts will reveal the effective damping performance and fast settling time exerted by the MMPSC based controller compared to conventional AVR and ANN based controller in Table 2.

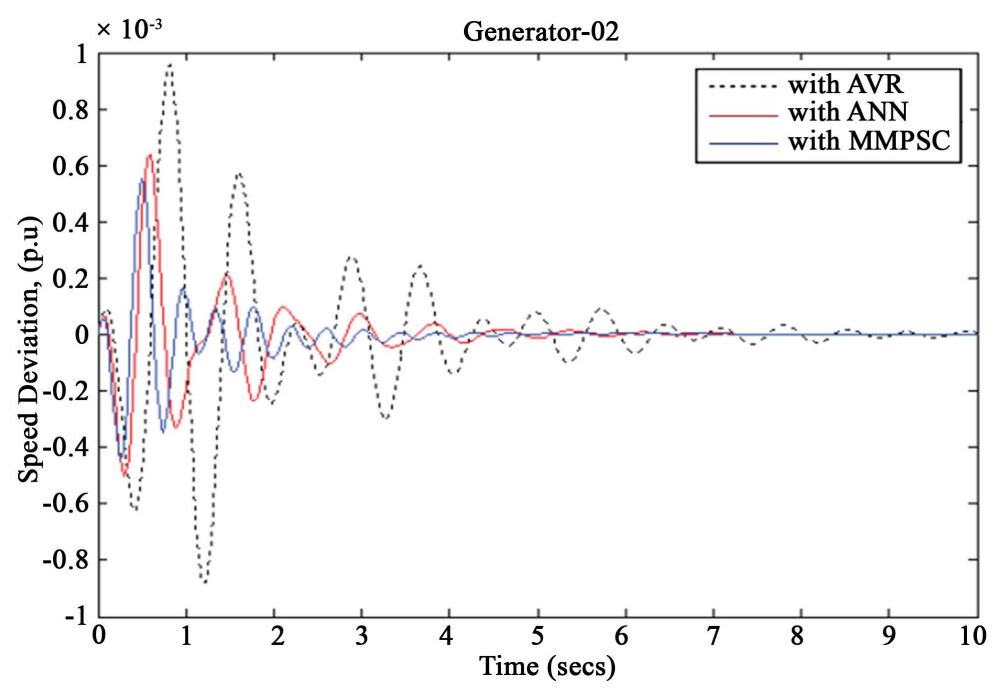

Figure 8. Speed deviation responses of $\mathrm{G} 2$ for $\mathrm{P}=0.24, \mathrm{Q}=0.055, \Delta \mathrm{Pd}=0.02$ p.u condition.

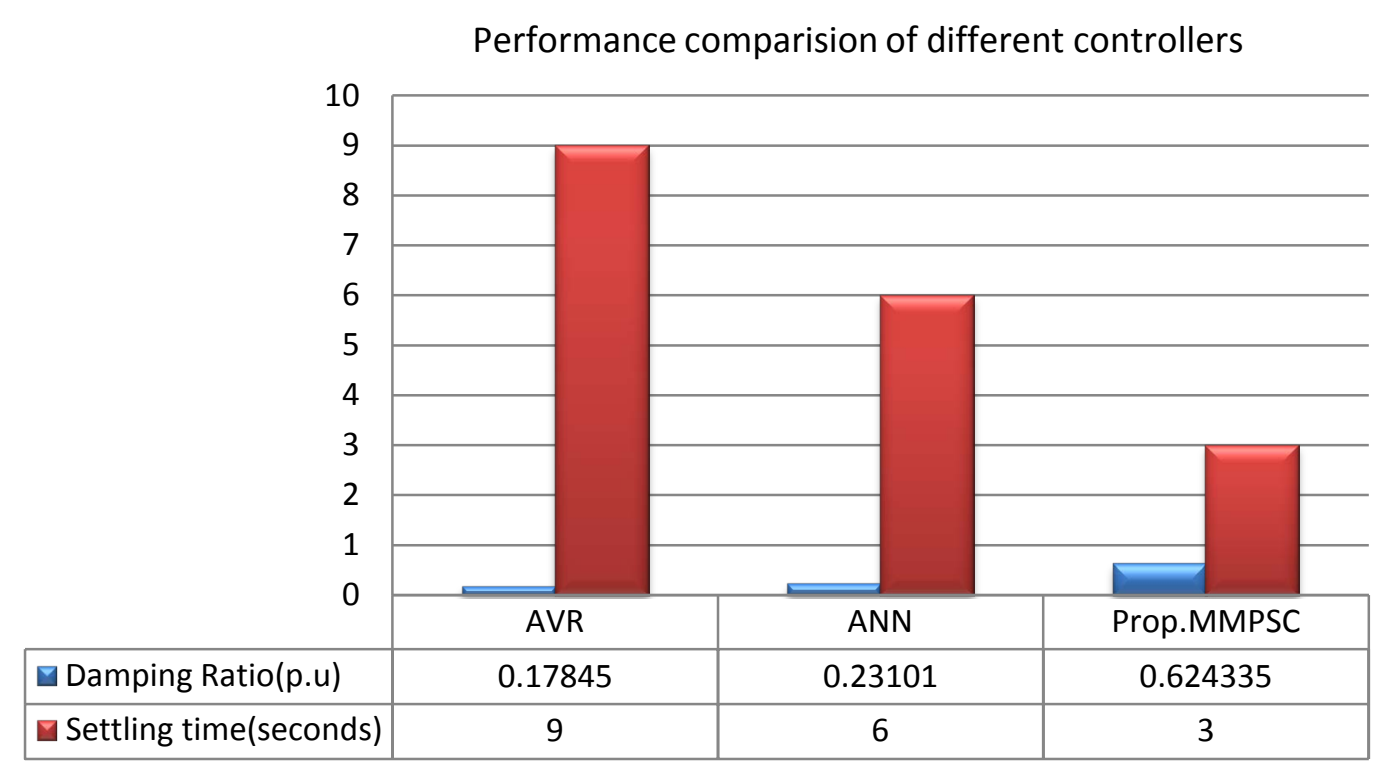

Figure 9. Comparison results of various controllers.

Table 2. Comparison table for stability analysis parameters.

\begin{tabular}{ccc}
\hline Controller Type & Damping Ratio (p.u.) & Settling Time in Seconds \\
\hline AVR & 0.17845 & 9 \\
ANN & 0.23101 & 6 \\
Prop. MMPSC & 0.624355 & 3 \\
\hline
\end{tabular}


The MMPSC with control algorithm is generating reference signal into produced compensating current to reduce the voltage sag and compensating reactive power at load side.

\section{Conclusion}

In this work a fast limited-time, nonlinear mode control of MMPSC is derived. The proposed control strategy applies nonlinear function to overcome stability problem and achieves fast unity power quality by accelerating rotor speed when the system is closed to the origin. Hence the proposed work implementation based on closed loop system stability analysis involved in the system models has been presented. The open loop system is unstable, and three damping controllers are implemented in the multi-ma- chine system to enhance the system stability. A detailed stability analysis has been presented, based on the computed eigen values, damping ratios and deviation responses of the system models. Based on the various closed loop stability analysis, it is evident that the proposed MMPSC is better than the conventional AVR and ANN based controller for multi-machine power system stability enhancement. Simulation results are provided to show the effectiveness and superiority of proposed control scheme. The proposed control method can be applied to secure communication and network synchronization.

\section{References}

[1] Chen, X.W., Zhang, W.N. and Zhang, W.D. (2005) Chaotic and Subharmonic Oscillations of a Nonlinear Power System. IEEE Transactions on Circuits and Systems II: Express Briefs, 52, 811-815.

[2] Ma, M.L. and Min, F.H. (2015) Bifurcation Behavior and Coexisting Motions in a Time-Delayed Power System. Chinese Physics B, 24, 030501. http://dx.doi.org/10.1088/1674-1056/24/3/030501

[3] Wei, D.Q. and Luo, X.S. (2009) Noise-Induced Chaos in Single-Machine Infinite-Bus Power Systems. Europhysics Letters (EPL), 86, 50008. http://dx.doi.org/10.1209/0295-5075/86/50008

[4] Chiang, H.D., Liu, C.W., Varaiya, P.P., Wu, F.F. and Lauby, M.G. (1993) Chaos in a Simple Power System. IEEE Transactions on Power Systems, 8, 1407-1417. http://dx.doi.org/10.1109/59.260940

[5] Wang, H.O., Abed, E.H. and Hamdan, A.M.A. (1994) Bifurcations, Chaos, and Crises in Voltage Collapse of a Model Power System. IEEE Transactions on Circuits and Systems I: Fundamental Theory and Applications, 41, $294-302$. http://dx.doi.org/10.1109/81.285684

[6] Lu, Q., Mei, S.W. and Sun, Y.Z. (2008) Power System Nonlinear Control. Tsinghua University Press, Beijing (in Chinese).

[7] Li, H.Q., Liao, X.F. and Liao, R.J. (2012) A Unified Approach to Chaos Suppressing and Inducing in a Periodically Forced Family of Nonlinear Oscillators. IEEE Transactions on Circuits and Systems I: Regular Papers, 59, 784-795,. http://dx.doi.org/10.1109/TCSI.2011.2169884

[8] Saad, M.S., Hassouneh, M.A., Abed, E.H. and Edris, A.A. (2005) Delaying Instability and Voltage Collapse in Power Systems Using SVCs with Washout Filter-Aided Feedback. Proceedings of the 2005 American Control Conference, Portland, 8-10 June 2005, 4357-4362. http://dx.doi.org/10.1109/acc.2005.1470665

[9] Harb, A.M. and Abdel-Jabbar, N. (2003) Controlling Hopf Bifurcation and Chaos in a Small Power System. Chaos, Solitons \& Fractals, 18, 1055-1063. http://dx.doi.org/10.1016/S0960-0779(03)00073-0

[10] Ginarsa, I.M., Soeprijanto, A. and Purnomo, M.H. (2013) Controlling Chaos and Voltage Collapse Using an ANFIS-Based Composite Controller-Static var Compensator in Power Systems. International Journal of Electrical Power \& Energy Systems, 46, 79-88. http://dx.doi.org/10.1016/j.ijepes.2012.10.005

[11] Wei, D.Q., Luo, X.S. and Qin, Y.H. (2011) Controlling Bifurcation in Power System Based on LaSalle Invariant Principle. Nonlinear Dynamics, 63, 323-329. http://dx.doi.org/10.1007/s11071-010-9806-3

[12] He, X.Y., Wang, Q.Y. and Yu, W.W. (2014) Finite-Time Containment Control for Second-Order Multiagent Systems under Directed Topology. IEEE Transactions on Circuits and Systems II: Express Briefs, 61, 619-623.

[13] Wu, Y.Q., Wang, B. and Zong, G.D. (2005) Finite-Time Tracking Controller Design for Nonholonomic Systems with Extended Chained Form. IEEE Transactions on Circuits and Systems II: Express Briefs, 52, 798-802.

[14] Polyakov, A. (2012) Nonlinear Feedback Design for Fixed-Time Stabilization of Linear Control Systems. IEEE Transactions on Automatic Control, 57, 2106-2110. http://dx.doi.org/10.1109/TAC.2011.2179869

[15] Cruz-Zavala, E., Moreno, J.A. and Fridman, L.M. (2011) 'Uniform Robust Exact Differentiator. IEEE Transactions on Automatic Control, 56, 2727- 2733. http://dx.doi.org/10.1109/TAC.2011.2160030

[16] Zuo, Z.Y. (2015) Nonsingular Fixed-Time Consensus Tracking for Second-Order Multi-Agent Networks. Automatica, 
54, 305-309. http://dx.doi.org/10.1016/j.automatica.2015.01.021

[17] Zuo, Z.Y. (2015) Non-Singular Fixed-Time Terminal Sliding Mode Control of Non-Linear Systems. IET Control Theory \& Applications, 9, 545-552. http://dx.doi.org/10.1049/iet-cta.2014.0202

[18] Erlich, I., Wrede, H. and Feltes, C. (2008) Dynamic Behaviour of DFIG-Based Wind Turbine During Grid Faults. IEEJ Transactions on Industry Applications, 128, 396-401. http://dx.doi.org/10.1541/ieejias.128.396

[19] Sun, T., Chen, Z. and Blaabjerg, F. (2005) Transient Stability of DFIG Wind Turbines at an External Short Circuit Fault. Wind Energy, 8, 345-360. http://dx.doi.org/10.1002/we.164

[20] Hasan, A.D. and Michalke, G. (2007) Fault Ride-Through Capability of DFIG Wind Turbines. Renewable Energy, 32, 1594-1610. http://dx.doi.org/10.1016/j.renene.2006.10.008

[21] Santos, S. and Le, H.T. (2007) Fundamental Time-Domain Wind Turbine Models for Wind Power Studies. Renewable Energy, 32, 2436-2452. http://dx.doi.org/10.1016/j.renene.2006.12.008

[22] Asao, T., Takahashi, R., Murata, T., Tamura, J., et al. (2007) Smoothing Control of Wind Power Generator Output by Superconducting Magnetic Energy Storage System. Proceedings of International Conference on Electric Machines and Systems, Seoul, 8-11 October 2007, 302-307.

\section{Submit or recommend next manuscript to SCIRP and we will provide best service for you:}

Accepting pre-submission inquiries through Email, Facebook, LinkedIn, Twitter, etc.

A wide selection of journals (inclusive of 9 subjects, more than 200 journals)

Providing 24-hour high-quality service

User-friendly online submission system

Fair and swift peer-review system

Efficient typesetting and proofreading procedure

Display of the result of downloads and visits, as well as the number of cited articles

Maximum dissemination of your research work

Submit your manuscript at: http://papersubmission.scirp.org/ 